\title{
CONSEJERÍA PSICOLÓGICA VIRTUAL EN LA UNIVERSIDAD COLOMBIANA: MÁS ALLÁ DEL RENDIMIENTO ACADÉMICO
}

Germán Andrés Torres Escobar', Laura Stella Parra²

\section{Resumen}

El servicio de consejería psicológica que se ofrece desde el bienestar universitario a los programas educativos virtuales en Colombia se ha centrado principalmente en detectar estudiantes con bajo rendimiento académico y con riesgo de deserción. Por esta razón, se han dejado un poco de lado los problemas psicológicos de los estudiantes, tales como la ansiedad académica, el desgaste emocional que implica trabajar y estudiar, y la prevención de situaciones de crisis que, eventualmente, se podrían presentar. Desde esta perspectiva, el presente artículo plantea una reflexión acerca de los nuevos retos del psicólogo que trabaja en la consejería psicológica virtual de las universidades y de la necesidad que tiene de formarse en el manejo de plataformas virtuales y la importancia de una regulación ética de sus acciones profesionales, de acuerdo con los lineamientos de la American Psychological Association, la Canadian Psychological Association y la New Zealand Psychologists Board.

Palabras clave: consejería virtual, telepsicología, acompañamiento, orientación, principios éticos

${ }^{1}$ Psicólogo, Magíster en Pedagogía y Magister en Psicología Educativa, docente investigador de tiempo completo de la Facultad de Psicología de la Universidad Católica Luis Amigó. Correo electrónico: german.torreses@amigo.edu.co, Código Postal 111121.

${ }^{2}$ Licenciada en Ciencias Sociales, Magíster y Doctorante en Educación, docente investigadora de medio tiempo de Facultad de Psicología de la Universidad Católica Luis Amigó. Correo electrónico: laura.parraes@amigo.edu.co, Código Postal 111121. 


\title{
VIRTUAL PSYCHOLOGICAL COUNSELING IN THE COLOMBIAN UNIVERSITY: BEYOND THE ACADEMIC PERFORMANCE
}

\author{
Germán Andrés Torres Escobar', Laura Stella Parra²
}

\section{Abstract}

The psychological counseling service offered by university welfare to virtual educational programs in Colombia, has focused mainly on detecting students with low academic performance and risk of dropping out, leaving aside the psychological problems of students such as academic anxiety, emotional strain caused by working and studying, as well as the prevention of situations of crisis, which could eventually arise. From this perspective, this article proposes a reflection on the new challenges faced by psychologist working in the virtual psychological counseling of the universities, and their need to be trained to use virtual platforms and the importance of an ethical regulation of their professional performance in accordance with the provisions of the American Psychological Association, the Canadian Psychological Association and the New Zealand Psychologists Board. 


\section{ACONSELHAMENTO PSICOLÓGICO VIRTUAL NA UNIVERSIDADE COLOMBIANA: ALÉM DO DESEMPENHO ACADÊMICO}

Germán Andrés Torres Escobar ${ }^{1}$, Laura Stella Parra²

\section{Resumo}

O serviço de aconselhamento psicológico oferecido pela previdência universitária a programas educacionais virtuais na Colômbia tem se concentrado principalmente na detecção de alunos com baixo desempenho acadêmico e risco de abandono, deixando de lado os problemas psicológicos dos alunos, como a ansiedade acadêmica, o desgaste emocional que envolve trabalhar e estudar, bem como a prevenção de situações de crise, que podem eventualmente surgir. Nessa perspectiva, este artigo propõe uma reflexão sobre os novos desafios do psicólogo que atua no aconselhamento psicológico virtual das universidades e a necessidade de capacitação na gestão de plataformas virtuais e a importância de uma regulação ética de suas ações profissionais de acordo com as diretrizes da American Psychological Association, da Canadian Psychological Association e da New Zealand Psychologists Board. 


\section{Introducción}

El presente artículo pretende hacer una reflexión acerca de la consejería psicológica virtual como alternativa de respuesta frente a las necesidades psicológicas de los universitarios que se matriculan en carreras virtuales.

En primer lugar, la consejería ${ }^{1}$ en línea es un área que en Colombia está expandiéndose gradualmente en las universidades $^{1}(1)$ a través del servicio de bienestar universitario $(2,3,4)$ y que se centra principalmente en identificar y atender estudiantes con bajo rendimiento en sus estudios, para evitar la deserción o pérdida del periodo académico(5).

Actualmente, cada institución de educación superior maneja su propia plataforma virtual $(6,7)$ en la que se encuentran cursos y planes de estudio, y se desarrollan actividades académicas dirigidas a los estudiantes. Por ello, cuando un estudiante no ingresa a la plataforma con frecuencia, no participa en las actividades académicas sugeridas o presenta notas bajas, se identifica como un estudiante en riesgo de deserción $(8,9)$

Aunque hay múltiples causas por las cuales un estudiante puede llegar a desertar de un programa académico virtual, estas pueden ser de tipo académico y extracadémico(10,11) Dentro ${ }^{1}$ El concepto de consejería ha sido definido por la American Psychological Association (APA), como un área especializada encargada de brindar asesoría, orientación y acompañamiento a las personas en temas relativos a la salud, elección de carrera y toma de decisiones en general. Dicha definición está disponible en la página web: http://www.apa. org/ed/graduate/specialize/counseling.aspx de las causas de tipo académico, están la falta de hábitos de estudio adecuados, las dificultades para gestionar el tiempo personal y el bajo nivel de preparación académica previa para responder a las exigencias de su carrera(). En las causas de tipo extracadémico, están la ansiedad académica, los problemas familiares o laborales que indirectamente pueden influir en la motivación académica del estudiante, las dificultades económicas para costear los estudios y las falencias para manejar crisis de tipo personal $(13,14)$.

De igual manera, es importante considerar el hecho de que el estudiante que ingresa a programas de educación superior virtual tiene un perfil diferente al del estudiante de la educación superior presencial. En efecto, generalmente el estudiante de carreras virtuales es una persona cercana a la adultez media, que trabaja para pagar sus estudios y que se vincula a la carrera porque tiene propósitos claros y, en ciertos casos, que procede de zonas rurales o apartadas de las grandes ciudades $(15,16)$.

Todas estas condiciones involucran las posibles causas de deserción y las características del perfil del estudiante de carreras virtuales, $y$ hacen que requiera no solo apoyo por parte de sus docentes a nivel académico, sino también de los profesionales de bienestar universitario, quienes, según el artículo 117 de la Ley 30 de 1992(17), deben desarrollar actividades orientadas a promover el desarrollo físico, psicoafectivo, espiritual y social de los estudiantes.

Por esta razón, el área de bienestar universitario de programas virtuales suele emplear estrategias como el contacto te- 
lefónico o electrónico(18). con los estudiantes, que permite indagar las causas de su bajo rendimiento académico o bajo nivel de participación, y posteriormente brindarles talleres y sesiones de consejería académica o psicológica que les permita superar tales dificultades y progresar en sus estudios. Otras estrategias son ofertar cursos de nivelación o vacacionales que permitan mejorar sus competencias lógico-matemáticas o de lectura crítica, desarrollar talleres de hábitos de estudio, consejerías personalizadas, etc.

Sin embargo, cabe aclarar que, debido al volumen de estudiantes que presentan dificultades académicas y personales, en ocasiones el tiempo y el número de profesionales de bienestar universitario son insuficientes para abordar a profundidad los problemas de tipo psicológico, familiar y emocional de los estudiantes de programas virtuales. Por ello, no siempre es posible dar una respuesta efectiva a este tipo de necesidades(19). Como aporte a dicho fenómeno, a continuación se exponen algunas de las principales soluciones que tienen los profesionales de bienestar universitario para resolver efectivamente tanto el abordaje de problemas psicológicos que pueden afectar el desempeño académico de los estudiantes de programas virtuales, como la cobertura de toda la población estudiantil en línea que presenta necesidad de apoyo psicológico.

\section{Solución 1. Conformación de comuni- dades de aprendizaje virtuales}

Cuando un estudiante tiene problemas psicológicos de origen familiar y personal que le dificultan desempeñarse bien en sus estudios, tiende a sentirse solo y a aislarse de las personas en la medida en que se sienta incomprendido. El problema se puede llegar a acentuar en programas de educación virtual, donde la interacción entre las personas está enfocada en temas académicos y no se dan espacios para abordar temas de tipo personal(20).

Por esta razón, Engstrom y Tinto(21) afirman que una de las razones por las cuales un estudiante puede llegar a retirarse de su carrera es la dificultad para vincularse social y académicamente con la institución educativa. Así, proponen que se construyan comunidades de aprendizaje que promuevan la integración de los estudiantes entre ellos y con la institución educativa. Una manera de construir comunidades de aprendizaje en la actualidad sería mediante el uso de herramientas virtuales(22) como, por ejemplo, plataformas y/o redes sociales que cuenten con un moderador o líder de bienestar universitario virtual por cada carrera o programa virtual. Esta persona estaría encargada de invitar a los estudiantes a vincularse académica y personalmente, indagar por sus necesidades de apoyo, plantear actividades de integración alrededor de los intereses o necesidades de los estudiantes y hacer un proceso de acompañamiento a lo largo de su trayectoria académica(23).

En relación con el elevado número de estudiantes que pertenecen a los programas virtuales frente al número de profesionales de bienestar universitario virtual, la idea que se propone es que los psicólogos líderes de cada carrera se encarguen de la conformación de mi- 
croredes de contacto con los estudiantes por semestre. De este modo, se forman pequeñas comunidades de aprendizaje en las que sus miembros puedan llegar a conocerse bien, interactuar y desarrollar actividades de formación personal y vinculación. Tales microredes pueden organizarse, eventualmente, en grupos del mismo semestre o periodo académico que viven en una misma región o bajo otra modalidad.

En la medida en que el líder de bienestar universitario virtual realice un seguimiento más cercano y detallado del grupo década microred de estudiantes, será posible detectar oportunamente en cada carrera los factores de riesgo potencial que podrían llevar a los estudiantes a la deserción. El objetivo de las microcomunidades de aprendizaje, entonces, es establecer vínculos cercanos con y entre los estudiantes, de una manera informal que permita abordar los diversos aspectos de sus vidas que influyen en su esfera académica(24). Por ejemplo, sería adecuado suscitar espacios para escuchar cómo manejan su tiempo, si perciben respaldo por parte de su familia para estudiar y si se sienten apoyados por los docentes y compañeros que hacen parte de la carrera en la cual están matriculados.

A su vez, este tipo de vinculación cercana podría ser de utilidad para evaluar con mayor precisión el nivel de satisfacción estudiantil y de calidad de los servicios universitarios, con miras a generar procesos de cambio y mejoramiento respecto a los servicios que ofrece el bienestar universitario virtual, los docentes de las carreras virtuales y la universidad, a nivel de acceso a herramientas tecno- lógicas, alternativas de financiación, entre otras.

\section{Solución 2. Consejería psicológica vir- tual personalizada}

Si al interior de las microcomunidades de aprendizaje antes mencionadas se detectan casos puntuales de estudiantes que presentan riesgo de desertar de sus estudios, se hace necesario implementar estrategias que permitan que los profesionales de bienestar universitario virtual ofrezcan un acompañamiento personalizado y en tiempo real, uno que dé respuesta oportuna y humana a las dificultades del estudiante.

Lograrlo no es sencillo, por lo que se debe sensibilizar a los estudiantes de los beneficios de este acompañamiento personalizado(25), la elaboración de protocolos de evaluación virtual, el diseño de protocolos de intervención virtual en crisis(26) y evaluar la efectividad de las prácticas de consejería virtual $(27,28)$

Asimismo, este servicio de consejería psicológica personalizada debe fundamentarse en la detección de alertas tempranas(29) o en reportes de casos por parte de docentes y directivos de cada carrera virtual. Así, el psicólogo podrá identificar indirectamente estudiantes con problemas emocionales o familiares e invitarlos a participar de encuentros virtuales para se pueda evaluar con mayor profundidad tales necesidades, darles orientaciones y hacer seguimiento.

Desde esta perspectiva, es esencial que el psicólogo de bienestar universitario virtual capacite a los docentes y directivos 
de cada facultad o programa académico acerca de los servicios de consejería psicológica de la institución educativa, la importancia de reportar al psicólogo casos de estudiantes con dificultades académicas y psicológicas que se identifiquen en las clases, y el vínculo humano entre docente-estudiante para generar confianza, un buen clima educativo y trabajar en pro de la formación integral del ser humano.

\section{Solución 3. Formación de psicólogos de bienestar universitario virtual en consejería virtual y cibersicología}

Los psicólogos de bienestar universitario virtual requieren capacitarse en consejería virtual y cibersicología para ofrecer un mejor servicio y mejorar sus prácticas de evaluación e intervención. Muchos psicólogos profundizan en campos específicos de la psicología, como el educativo o clínico, para trabajar en bienestar universitario. Sin embargo, en la formación disciplinar que ofrecen dichos programas, generalmente, no abarca el tema de diseño de contenidos en línea, prácticas de evaluación e intervención en ciberpsicología y aspectos éticos de esta modalidad. Por ejemplo, dentro de los contenidos curriculares de la formación de psicólogos que establece la Resolución 3461 de 2003()no se mencionan contenidos referentes a la evaluación o desarrollo de intervenciones en línea. El mismo caso sucede con la Ley 1090 de 2006(31).

Lo mencionado previamente se constituye en un reto para los programas de formación de posgrado en psicología, ya que es muy probable que en un fu- turo cercano los psicólogos se vean en la necesidad de desarrollar contenidos digitales(32) referentes a evaluaciones psicológicas, procesos de acompañamiento, procesos terapéuticos, capacitaciones de personal e investigaciones en línea(33).

Por ende, los programas de pregrado y posgrado en psicología presencial y virtual deberían incluir dentro de sus planes de estudio, cursos de evaluación e intervención en línea, abordaje de dilemas éticos en línea. Por otro lado, a nivel de formación continua, el Colegio Colombiano de Psicólogos podría ofrecer cursos y diplomados en temas como el diseño de contenidos en plataformas virtuales, manejo de la confidencialidad de la información en entornos virtuales y las estrategias de comunicación digital.

El objetivo de este tipo de actividades es que los psicólogos de bienestar universitario virtual tengan una aproximación al campo de la consejería virtual, comprendan sus principales ejes temáticos e incursionen en esta área tanto a nivel práctico como investigativo(34). Respecto a esta idea, en la Tabla 1 se muestran ejes temáticos como las evaluaciones psicológicas en línea y sus características, procesos de acompañamiento en línea, capacitaciones en línea e investigaciones en línea. Cada uno de estos ejes debe plantarse a partir de las necesidades de vinculación académica y social que se detecten en los estudiantes al interior de las microcomunidades de aprendizaje.

En primera instancia, las evaluaciones 
aspectos, tales como el manejo de la confidencialidad, la gestión de la información y las restricciones de acceso a la misma(35). A nivel de los procesos de acompañamiento y orientación, hay que considerar sus alcances y limitaciones, dependiendo de la gravedad de los problemas psicológicos de la persona a la que se va a atender.

En lo concerniente a las capacitaciones grupales de personas, podría abrirse la posibilidad de conectar el trabajo de consejería psicológica online con actividades de extensión académica en las universidades a través de cursos de formación personal que den respuesta a las necesidades de personas de acuerdo con su localización geográfica. Su efectividad se evaluaría con base en los resultados de tales cursos y procesos de acompañamiento virtual.

Tabla 1. Ejes temáticos en consejería universitaria virtual y sus problemáticas

\begin{tabular}{|c|c|}
\hline Eje temático & Problemáticas por abordar \\
\hline $\begin{array}{l}\text { Evaluaciones } \\
\text { psicológicas } \\
\text { en línea }\end{array}$ & $\begin{array}{l}\text { - Manejo confidencial de documentos digitales con claves de } \\
\text { - } \quad \text { Feguridad. } \\
\text { - } \quad \text { Consentimientos informados digitales. } \\
\text { - } \quad \text { Evaluaciones cualitativas digitales. } \\
\text { - } \quad \text { Gestión de bases de datos de evaluaciones psicológicas en línea. } \\
\text { - } \quad \text { Restricciones de acceso a informes de evaluación psicológica en } \\
\quad \text { línea. }\end{array}$ \\
\hline $\begin{array}{l}\text { Procesos de } \\
\text { acompañamiento y } \\
\text { orientación en línea }\end{array}$ & $\begin{array}{l}\text { - Restricciones en horarios de acompañamiento y orientación en } \\
\text { línea. } \\
\text { - Acogida y manejo de intervenciones en crisis en línea ante } \\
\text { situaciones de ideación suicida, episodios depresivos agudos, } \\
\text { episodios psicóticos agudos o denuncias por violencia } \\
\text { intrafamiliar. } \\
\text { - Diseño de guías virtuales de orientación en toma de decisiones. } \\
\text { - Procesos de orientación profesional en línea. }\end{array}$ \\
\hline $\begin{array}{c}\text { Capacitaciones en } \\
\text { línea }\end{array}$ & $\begin{array}{l}\text { - } \quad \text { Detección de necesidades grupales de capacitación en línea. } \\
\text { - Ejercicios de aprendizaje de habilidades sociales y competencias } \\
\text { blandas basados en simulación virtual. }\end{array}$ \\
\hline $\begin{array}{l}\text { Investigaciones en } \\
\text { línea }\end{array}$ & $\begin{array}{l}\text { - Confidencialidad y veracidad de denuncias en línea. } \\
\text { - Ciberacoso: estrategias de detección y manejo (Trujano, Dorantes } \\
\text { - ETovilla, 2009). } \\
\text { - } \text { línectividad y resultados obtenidos en prácticas psicológicas en }\end{array}$ \\
\hline
\end{tabular}


Solución 4. Regulación de prácticas de consejería psicológica virtual a nivel nacional

Es necesario que en Colombia se diseñen unos lineamientos y regulaciones legales a futuro en torno al tema de la consejería psicológica virtual, para delimitar lo que se consideran buenas prácticas en esta modalidad y poder así juzgar conforme a estas las conductas profesionales virtuales. Por ende, hay que revisar estándares éticos de evaluación e intervención virtual que ya existen en otros lugares del mundo, como los de la American Psychological Association, la Canadian Psychological Association y la New Zealand Psychologists Board $(33,36,37)$, para que así se constituyan en pilares fundamentales para el desarrollo de estándares ético-legales en consejería virtual psicológica nacional.

\section{Conclusiones}

La consejería psicológica que se ofrece desde bienestar universitario para programas virtuales se ha enfocado principalmente en ayudar a estudiantes con bajo rendimiento académico que estén en riesgo de deserción. Por esta razón, la variables netamente académicas tienen mayor prioridad, por lo que se dejan en segundo plano las variables psicológicas que puedan afectar el desempeño del estudiante, a la vez que no se logra dar cobertura completa a todos los estudiantes que tienen una necesidad de apoyo.

En este sentido, a lo largo del artículo se propusieron cuatro soluciones que permitan a los profesionales que trabajan en bienestar universitario virtual dar respuesta efectiva a las necesidades psicológicas de los estudiantes y brindar un mayor impacto en toda la comunidad virtual de estudiantes. Son las siguientes:

La conformación de comunidades de aprendizaje virtual, lideradas por el psicólogo y que fomenten la integración social y académica de los estudiantes entre sí y con la institución educativa.

La oferta de servicios de atención psicológica personalizada en línea a aquellos estudiantes que presentan problemas psicológicos.

La formación y capacitación de los psicólogos de bienestar universitario virtual, para que puedan mejorar sus prácticas de evaluación e intervención virtual.

Generar estándares éticos y regulaciones legales que orienten a los profesionales de bienestar universitario virtual en la toma de decisiones propias de su área. 


\section{Referencias}

1. Pineda E, Meneses T, Téllez F. Análisis de redes sociales y comunidades virtuales de aprendizaje: Antecedentes y perspectivas. Revista virtual de la Universidad Católica del Norte. 2013;38:40-55.

2. Sánchez G. Bienestar en redes sociales. Su utilidad en educación virtual y a distancia. PSIENCIA-Revista Latinoamericana de Ciencia Psicológica. 2013;8(2):1-13.

3. Perdomo M, Cañón V. Bienestar universitario virtual, una estrategia para el fortalecimiento de la comunidad unadista con el uso de las TICs. Revista de Investigaciones UNAD Bogotá. 2011;2:109-25.

4. Llinás E. La orientación académica desde el bienestar universitario. Colombia: Ediciones Uninorte. 2009.

5. Zárate-Rueda R, Hernández-Suárez $M$. Orientaciones socialmente responsables para la permanencia estudiantil en programas de educación a distancia de la Universidad Industrial de Santander-UIS. Entramado. 2016;12(1):250-64.

6. Menéndez A y Sánchez C. Uso de plataformas social media en la práctica docente universitaria: investigación biográfico-narrativa en un estudio de caso. Signo y Pensamiento. 2013;32(63):152-68.

7. Ardila-Rodríguez M. Indicadores de calidad de las plataformas educativas digitales. Educación y Educadores.2011;14(1):189-206.

8. Arraiga J, Burrillo V, Carpeño A, Casaravilla A. Caracterización de los tipos de abandono. Dividamos el problema y venceremos más fácilmente. Ponencia presentada en la I Conferencia Latinoamericana sobre el Abandono en la Enseñanza Superior (I-CLABES) en Nicaragua. 2011. Disponible en: http://www.

122 alfaguia.org/www-alfa/images/ponencias/ clabesI/ST_1_Abandono/7_JArriaga_ Perfiles\%20abandono.pdf
9. Gómez S, Montero L. Estudiar carreras universitarias en modalidades e- learning y b- learning. Revista Lasallista de Investigación. 2015;12(2):94-104.

10. La Madriz J. Factores que promueven la deserción del aula virtual. Orbis. Revista Científica Ciencias Humanas. 2016;12(35):18-40.

11. Murcia N, Ramírez P. Motivos de deserción estudiantil en programas virtuales de posgrado: revisión de caso y consideraciones desde el mercadeo educativo y el mercadeo relacional para los programas de retención. Revista de Educación a Distancia. 2015;45:1-23.

12. Rugeles $P$, Mora B, Metaute P. El rol del estudiante en los ambientes educativos mediados por las TIC. Revista Lasallista de Investigación. 2015;12(2):132-8.

13. Areth J, Castro-Martínez J, Rodríguez, H. La educación virtual en Colombia: exposición de modelos de deserción. Apertura. 2015;7(1):1-10.

14. Serrano C, Rojas A, Ruggero C. Depresión, ansiedad y rendimiento académico en estudiantes universitarios. Revista Intercontinental de Psicología y Educación. 2013;15(1): 47-60.

15. Uribe R. El estudiante de educación virtual: proyección o reflexión. Apertura. 2008;8(9): 51-67.

16. Camacho I, Gómez M, Pintor M. Competencias digitales en el estudiante adulto trabajador. Revista Interamericana de Educación de Adultos. 2015;37(2):10-24.

17. Congreso de Colombia. Ley 30. Por la cual se organiza el servicio público de la Educación Superior. 1992. Disponible en: https://www.mineducacion.gov.co/1621/ articles-86437_Archivo_pdf.pdf 
18. Meilman P. "Wait! can you do this too?!?" College Counseling and Distance Education. Journal of College Student Psychotherapy. 2016;30(1):1-3.

19. Contecha L, Jaramillo L. El bienestar universitario subordinado a una modernidad instrumental. Revista Actualidad \& Divulgación Científica. 2011;14(1):101-109.

20. Rincón M. Los entornos virtuales como herramientas de asesoría académica en la modalidad a distancia. Revista virtual Universidad Católica del Norte. 2008;25.

21. Engstrom C, Tinto V. Access without support is not opportunity. Change. 2008;40(1):46-50.

22. Torres-Díaz J, Jara D, Valdiviezo P. Integración de redes sociales y entornos virtuales de aprendizaje. Revista de Educación a Distancia. 2013;35:1-8.

23. Meirinhos M, Osorio A. Las comunidades virtuales de aprendizaje: el papel central de la colaboración. Pixel-Bit. Revista de Medios y Educación. 2009;35:45-60.

24. Haberstroh, S. College Counselors' Use of Informal Language Online: Student Perceptions of Expertness, Trustworthiness, and Attractiveness.

Cyberpsychol Behav Soc Netw. 2010;13(4),455-59.

25. Pruitt L, Luxton D, Shore, P. Additional clinical benefits of home-based telemental health treatments. Professional Psychology: Research and Practice. 2014;45:340-6.

26. Da Silva J, Siegmund G, Bredemeier J. Crisis interventions in online psychological counseling. Trends Psychiatry Psychother. 2015;37(4):171-82.

27. Richards D, Viganó N. Online counseling: A narrative and critical Review of the Literature. Journal of Clinical Psychology. 2013;69(9):994-1011.

28. Chester A, Glass C. Online counselling: a descriptive analysis of therapy services on the Internet. $\mathrm{Br} \mathrm{J}$ Guid Counc. 2006;34(2):145-160.

29. Beltrán J. Sistema de alertas tempranas para la identificación de bajo rendimiento, pérdida de la calidad y seguimiento estudiantil, a través de tecnología web 2.0, en el programa de ingeniería de sistemas presencial de la Universidad de Cartagena (tesis). Cartagena: Universida de Cartagena; 2016.

30. Ministerio de Educación Nacional. Resolución 3461. Por la cual se definen las características específicas de calidad para los programas de pregrado en Psicología. Bogotá, D.C.: Ministerio de Educación. 2003.

31. Ministerio de Protección Social. Ley 1090. Por la cual se reglamenta el ejercicio de la profesión de Psicología, se dicta el Código Deontológico y Bioético y otras disposiciones. Bogotá, D.C.: Ministerio de protección social. 2006.

32. Maheu M, Pulier M, McMenamin J, Posen L. Future of Telepsychology, Telehealth, and Various Technologies in Psychological Research and Practice. Professional Psychology Research and Practice. 2012;43(6):613-21.

33. American Psychological Association. Guidelines for the practice of telepsychology. American Psychologist. 2013;68(9):791-800.

34.Mallen M, Vogel D, Rochlen A. The Practical Aspects of Online Counseling: Ethics, Training, Technology, and Competency. The Counseling Psychologist. (2013);33(6):776-818.

35. Turvey C, et al. ATA Practice Guidelines for Video-Based Online Mental Health Services. Telemedicine and e-Health. 2013;19(9):721-30.

36. New Zealand Psychologists Board [Internet]. Draft guidelines: Psychology services delivered via the Internet and other electronic media. 2011. Disponible en: 
INVESTIGACIONES ANDINA No. 38, Vol. 21

http://psychologistsboard. org.nz/cms show_download. php?id=141

37. Canadian Psychological Association [Internet]. Ethical guidelines for psycholo- gists providing psychological services via electronic media. (2006). Disponible en: http://www.cpa.ca/aboutcpa/committees/ ethics/psychserviceselectronically/. 